Revue des patrimoines

\title{
Transferts méthodologiques 3D appliqués à l'étude de l'art paléolithique : une nouvelle dimension pour les relevés d'art préhistorique
}

Methodological 3D transfers applied to the study of Palaeolithic art, new dimensions in recording prehistoric art

Oscar Fuentes, Julie Lepelé et Geneviève Pinçon

\section{(2) OpenEdition}

Journals

Édition électronique

URL : http://journals.openedition.org/insitu/21510

DOI : $10.4000 /$ insitu. 21510

ISSN : 1630-7305

Éditeur

Ministère de la Culture

Référence électronique

Oscar Fuentes, Julie Lepelé et Geneviève Pinçon, « Transferts méthodologiques 3D appliqués à l'étude de l'art paléolithique : une nouvelle dimension pour les relevés d'art préhistorique », In Situ [En ligne], 39 | 2019, mis en ligne le 24 mai 2019, consulté le 11 juillet 2019. URL : http:/l journals.openedition.org/insitu/21510; DOI : 10.4000/insitu.21510

Ce document a été généré automatiquement le 11 juillet 2019.

\section{c) (†)}

In Situ Revues des patrimoines est mis à disposition selon les termes de la licence Creative Commons Attribution - Pas d'Utilisation Commerciale - Pas de Modification 4.0 International. 


\title{
Transferts méthodologiques 3D appliqués à l'étude de l'art paléolithique : une nouvelle dimension pour les relevés d'art préhistorique
}

\author{
Methodological 3D transfers applied to the study of Palaeolithic art, new \\ dimensions in recording prehistoric art
}

Oscar Fuentes, Julie Lepelé et Geneviève Pinçon

\section{Introduction}

1 L'étude de l'art pariétal préhistorique pose comme geste intellectuel et technique la réalisation de relevés afin de transformer le support en autant d'éléments d'information permettant d'éclairer telle ou telle problématique du chercheur. L'objectif du « relevé » est de rendre visibles et accessibles des données qui peuvent être "cachées ", souvent non visibles dans l'immédiat. L'intérêt de la démarche est donc multiple, tant scientifique que pour la diffusion de la connaissance auprès du plus grand nombre.

2 Henri Breuil, André Glory, l'abbé Amédée Lemozi, Léon Pales, Michel Lorblanchet, Gilles Tosello, Carole Fritz, Geneviève Pinçon, entre autres, sont autant de préhistoriens à avoir marqué de leur savoir-faire, la difficile approche des représentations paléolithiques, par leur travail de relevé. Comme le rappelait Léon Pales, « l'on n'identifie à coup sûr qu'un trait sur mille $»^{1}$. Parfois critiqué, le relevé n'a pas toujours été considéré comme un geste indispensable à l'étude de l'art rupestre par l'ensemble des chercheurs ayant travaillé sur ce sujet. André Leroi-Gourhan par exemple, pour son étude majeure sur l'art paléolithique occidental, précise qu'il n'y a pas eu recours, considérant que le relevé pouvait être une étape intermédiaire gênante pour la compréhension des grands 
ensembles : « Les relevés des œuvres par le dessin sont précieux, mais ne rendent compte de la réalité qu'à travers le filtre d'une main étrangère ${ }^{2}$.»

3 Le relevé n'est assurément pas une pâle copie des œuvres de nos lointains ancêtres sapiens. Il n'a pas vocation à remplacer ces images, vestiges de l'expression culturelle de ces sociétés. Il est une démarche essentielle mais terriblement difficile, qui permet de rendre compte des complexités et richesses des œuvres paléolithiques. Il traduit nos compréhensions de ces traces du passé avec nos outils contemporains. À l'instar de la fouille planimétrique, le relevé est incontournable pour l'étude des vestiges graphiques.

L'utilisation des techniques numériques, et notamment celle de la 3D appliquée à l'étude de l'art paléolithique, se place dans une continuité historique des méthodes et techniques. Ces techniques s'inscrivent non pas comme une finalité de l'étude mais comme un ensemble de processus explorés par les chercheurs. Comment s'inscrivent ces mutations et innovations techniques imposées par la 3D dans les pratiques de recherche en art paléolithique? Quels sont les liens entre un processus classique de relevé d'art pariétal ou mobilier et des processus nouveaux apportés par la 3D ?

5 C'est dans cet objectif que nous proposons des développements méthodologiques à des processus techniques 3D. Les usages des environnements numériques 3D que nous présentons se veulent comme des jalons accompagnant le mouvement entamé depuis la démocratisation des outils numériques.

\section{Le relevé d'art paléolithique : deux méthodes pour deux statuts de l'image différents}

6 Les techniques de relevé adoptées par les chercheurs vont de pair avec la méthode qu'ils emploient pour étudier la paroi et traduire ce qu'ils observent. L'enjeu est alors de savoir comment traduire ce qui est observé avec les techniques les plus adaptées. C'est ce que Patrice Flichy appelle le cadre de référence, regroupant le cadre d'usage (les manières de faire) et le cadre sociotechnique (pratiques en lien avec les techniques) $)^{3}$. Cette notion est importante car elle permet de mieux définir les pratiques du relevé au sein de la recherche.

Deux tendances globales conjointes et différentes peuvent être identifiées. Toutes les deux procèdent d'une analyse des supports et se veulent donc "analytiques ». D'une manière synthétique, nous qualifierons ces deux choix, l'un de relevé "analytique plastique ", l'autre de relevé " analytique technique ». Ces deux modes procèdent de deux pré-supposés distincts : d'un côté, une hiérarchisation de l'information (relevé plastique), et de l'autre, l'approche cartographique exhaustive (relevé technique).

Le "relevé plastique " s'appuie sur une démarche structurelle de hiérarchisation et simplification du rendu graphique afin de valoriser l'image alors que le «relevé technique » ne propose pas de sélection des informations mais une traduction exhaustive de l'ensemble des stigmates réunis par calques de lecture. Plutôt que la sélection et la mise en avant de l'image par un rendu esthétique, le relevé technique met en avant une approche cartographique de l'ensemble des traces ; l'image est mise au même niveau que les autres registres informatifs. Carole Fritz et Gilles Tosello font la même distinction entre les deux mouvements méthodologiques. Cependant, ils parlent plutôt de la nécessaire différence entre un «relevé de lecture " (l'approche plastique) et un « relevé technique $»^{4}$ que nous nommons « relevé archéologique ». 
9 Ces deux approches posent en fait la question de l'image et de son statut (social et scientifique) dans l'analyse d'une part et le traitement des informations recueillies d'autre part. Le relevé plastique pose la représentation comme l'entité centrale à prendre en compte, permettant l'interprétation. Le relevé technique inverse la dialectique et décide de partir du contexte de paroi (éléments naturels) pour aller vers la figure (éléments anthropiques), au final.

Quels que soient les principes théoriques, ces deux approches s'appuient sur un choix méthodologique identique devenu la règle: la technique du relevé indirect (par photographie, ortho-photographie ou vidéo-projection).

\section{Le relevé de type analytique plastique : la méthode dite classique}

11 Les relevés plastiques se basent sur un enregistrement hiérarchisé des éléments observés, un processus de traduction graphique des stigmates de paroi. Sur le site web du CREAP ${ }^{5}$, il est possible de lire :

L'objectif du relevé est de proposer au lecteur une interprétation graphique qui rende compte de la complexité des faits observés, soit un document complémentaire de la photographie. Cette fonction implique une indispensable et préalable réduction des données ; en d'autres termes, des choix doivent être opérés car le potentiel d'informations susceptibles d'être enregistrées sur la paroi est immense et l'accumulation risque de conduire à une confusion visuelle. Par exemple, les données caractérisant le support (calcite, fissures...) ne doivent pas gêner la perception des œuvres ${ }^{6}$.

12 Le principe est donc basé sur une démarche "graphique et plastique ». Le processus inscrit l'analyse de la paroi et de l'ensemble des données observées. La hiérarchisation se fait par un travail plastique (traitement informatique de l'image) des ensembles, avec donc une mise en avant de l'image.

13 L'impérieuse nécessité d'intégrer les données sur l'état de surface, sur sa conservation, fait que des éléments liés à l'état naturel apparaissent dans les rendus plastiques. Les relevés des parois ornées, comme à Marsoulas (Haute-Garonne) par exemple, au cours des années 1990, introduisent des notions d'évolution des états de parois au cours du temps pour expliquer les états conservatoires actuels, notamment en lien avec les nombreux graffitis modernes masquant les œuvres. Mais la recherche impose également d'intégrer des données complémentaires comme la morphologie de la paroi, les micro-topographies, le rapport entre surface naturelle, volume et image représentée (fig. 1). 
Figure 1

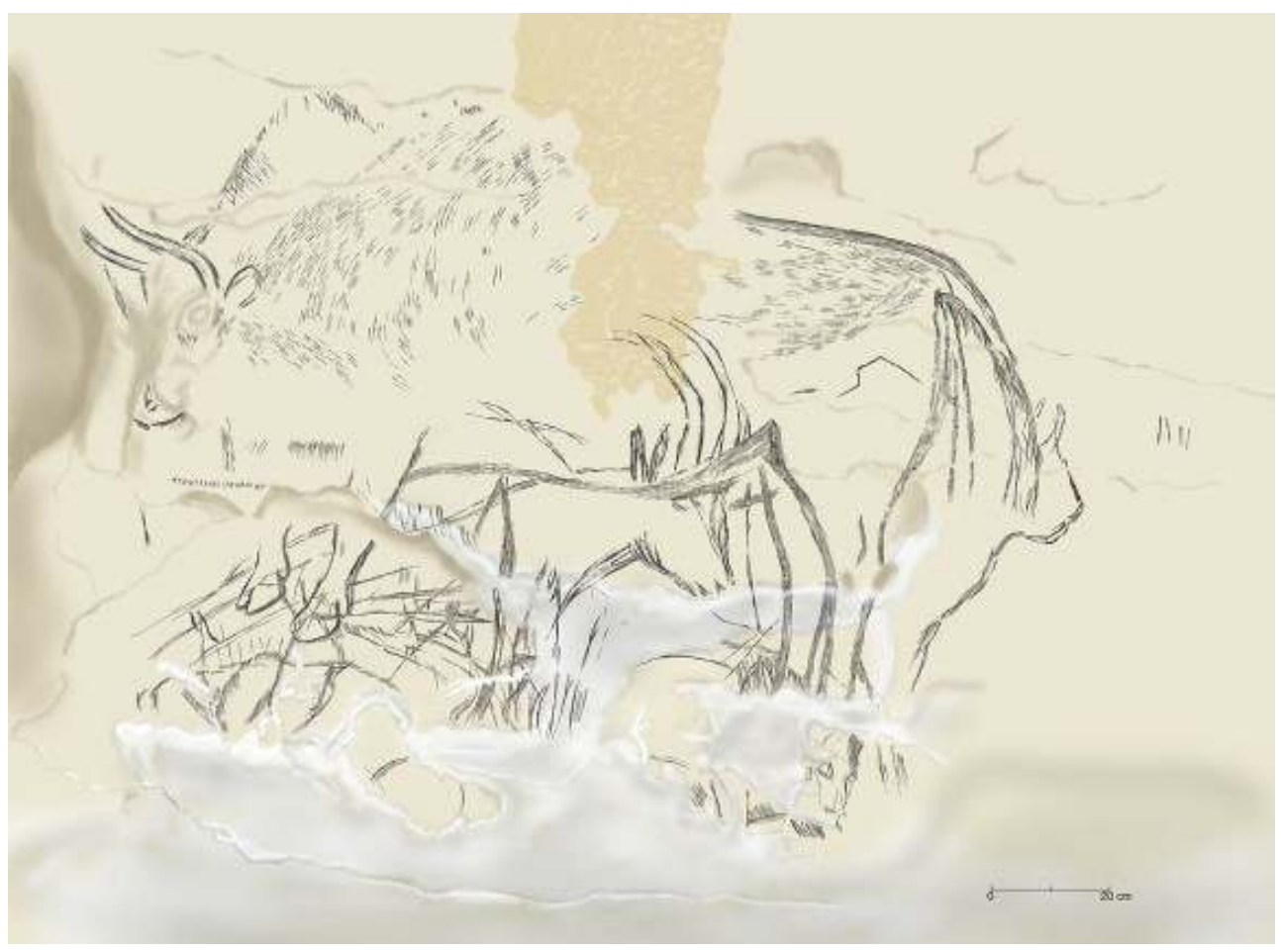

Relevé plastique, dessin et gravures pariétales de Marsoulas, Bison. Fritz et. al., 2010.

(c) Gilles Tosello et Carole Fritz.

14 Ce résultat est obtenu à la suite d'un processus méthodologique qui est aujourd'hui qualifié de « classique » ou «traditionnel » et dont les bases ont été publiées par Carole Fritz et Gilles Tosello en 2007

Les avantages de cette méthode sont multiples, comme celui de rendre accessibles l'image, le geste et les chronologies de réalisation. Cependant, elle s'appuie sur une primauté de l'image, elle crée une césure entre l'image représentée et le support, celui-ci étant souvent présenté comme fond plastique, soutien de l'iconographie. Nous retrouvons les préceptes mis en avant par Carole Fritz et Gilles Tosello sur les méthodes de relevé et résumés par Marc Azéma : «Bien que l'impact d'autres facteurs (comme la morphologie des supports, la présence de griffades) soit également pris en compte, une hiérarchie visuelle favorisant les manifestations anthropiques doit être privilégiée. Cela implique une indispensable et préalable sélection des données: en d'autres termes, des choix doivent être opérés ${ }^{8} . . . »$ (fig. 2). 
Figure 2

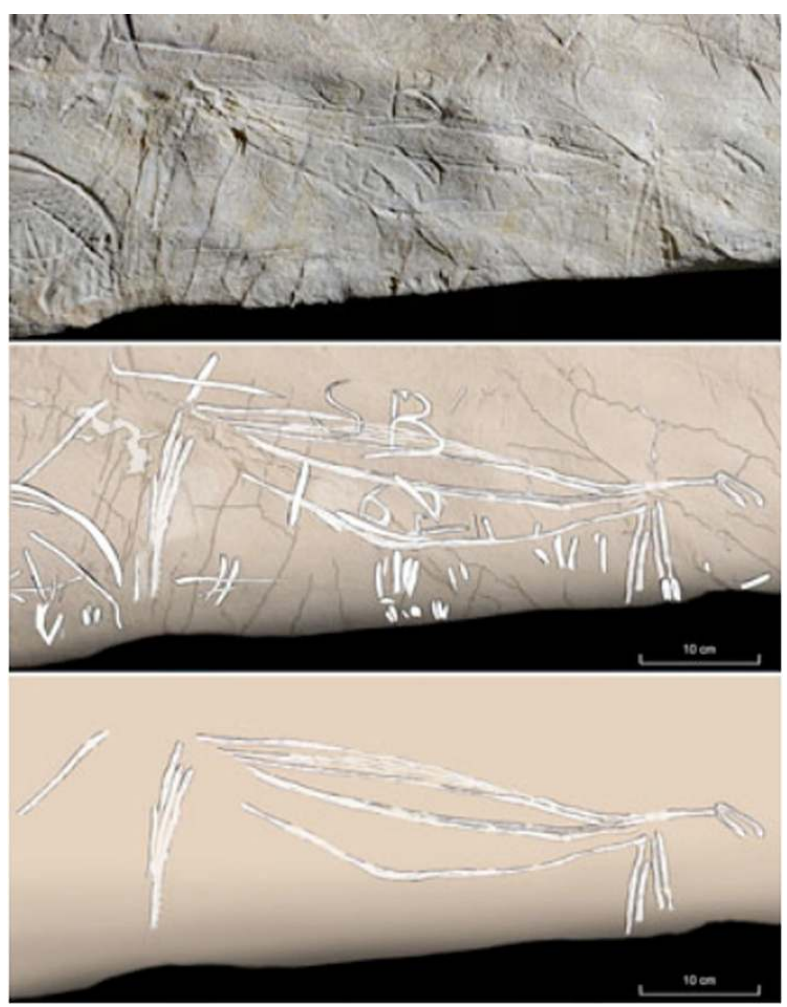

Schéma de sélection à partir de la méthode de relevé classique d'après photographie, félin gravé de la Salle Bégouën, grotte de la Baume-Latrone (Gard)

Relevé R. Bourillon et M. Azéma, 2012. (c) Azéma et. al.

Par ailleurs, la manifestation graphique étant l'élément central de l'étude, son traitement infographique trouve sa traduction logique dans les suites logicielles de type retouches d'images (comme Gimp pour le logiciel Open Source, ou la suite @AdobePhotoshop pour les licences privées). C'est avec ces environnements logiciels que les fonds de paroi sont tracés et traités.

\section{La méthode de type analytique archéologique : une cartographie pariétale vectorielle}

Par rapport au relevé plastique, le relevé archéologique («technique» inverse la dialectique de manière fondamentale. Ce n'est plus l'image qui est au cœur de la démarche mais le support portant les représentations. En conséquence de quoi, l'image paléolithique n'apparaitra qu'à la fin du relevé, comme le résultat d'un processus d'analyse et non comme la porte d'entrée de celle-ci. Dans la démarche, il n'y a d'ailleurs pas de croquis de lecture fixant au préalable une quelconque image.

Ce type de relevé s'est développé au cours de ces dernières décennies dans l'étude de l'art préhistorique et s'est construit notamment pour répondre aux problématiques particulières des grands ensembles ornés des abris sous roche sculptés. Cette méthode s'appuie sur une analyse fine de l'ensemble des stigmates de paroi (apports et enlèvements) distinguant s'ils sont d'origine naturelle ou anthropique, anciens ou récents. Les différentes informations relevées sur la paroi, observées par les chercheurs 
et qui sont destinées à être partagées, sont codées selon une charte graphique par l'association de motifs géométriques et de codes couleurs, à la manière d'une cartographie de surface ${ }^{9}$.

19 Cet enregistrement des traces observables pour répondre à des problématiques d'étude des œuvres pariétales a ouvert la voie à de multiples développements techniques et méthodologiques. La volonté de restituer les volumes a poussé les chercheurs à marquer les courbes de niveau, les reliefs naturels. Cela a conduit à penser l'image dans son contexte physique et sa localisation dans la cavité et à introduire la question de son état de conservation ${ }^{10}$.

20 C'est sur cette base que se sont orientées les méthodes et techniques développées notamment par Geneviève Pinçon et son équipe dans l'étude de la frise sculptée du Rocaux-Sorciers ${ }^{11}$ (Angles-sur-l'Anglin, Vienne) (fig. 3).

Figure 3

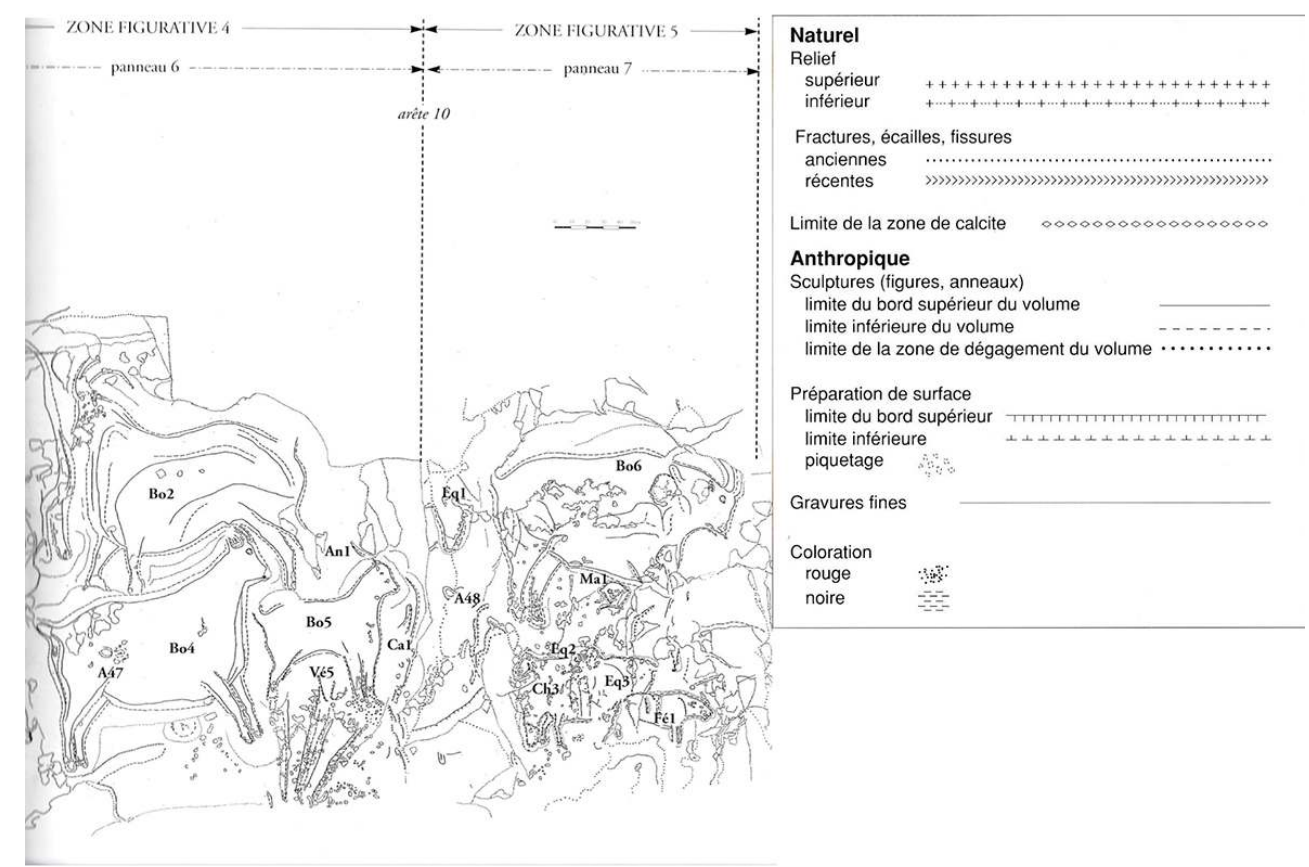

Relevé analytique exhaustif de la frise sculptée du Roc-aux-Sorciers (Vienne), panneau des Bouquetins, abri Bourdois avec légende de relevé.

Geneviève Pinçon et Ludmila lakovleva, 1997. ( C Geneviève Pinçon et Ludmila lakovleva.

21 Au début des années 2000, l'informatisation et la numérisation des relevés ont permis de procéder à leur vectorisation. Il a été créé autant de calques que de registres informatifs. Il a donc été possible de réaliser une bibliothèque de formes vectorielles ${ }^{12}$.

Ce procédé a facilité la mise en perspective des caractéristiques communes des frises sculptées du Roc-aux-Sorciers et de la Chaire-à-Calvin (Mouthiers-sur-Boëme, Charente) ${ }^{13}$ (fig. 4). Le relevé technique a aussi été employé pour les frises sculptées du Roc-de-Sers (Sers, Charente) ${ }^{14}$, de l'abri Reverdit (Sergeac, Dordogne) ${ }^{15}$, de Cap Blanc (Marquay, Dordogne $)^{16}$ et pour les décors pariétaux de Comarque (Eyzies-de-Tayac Sireuil) ${ }^{17}$. 


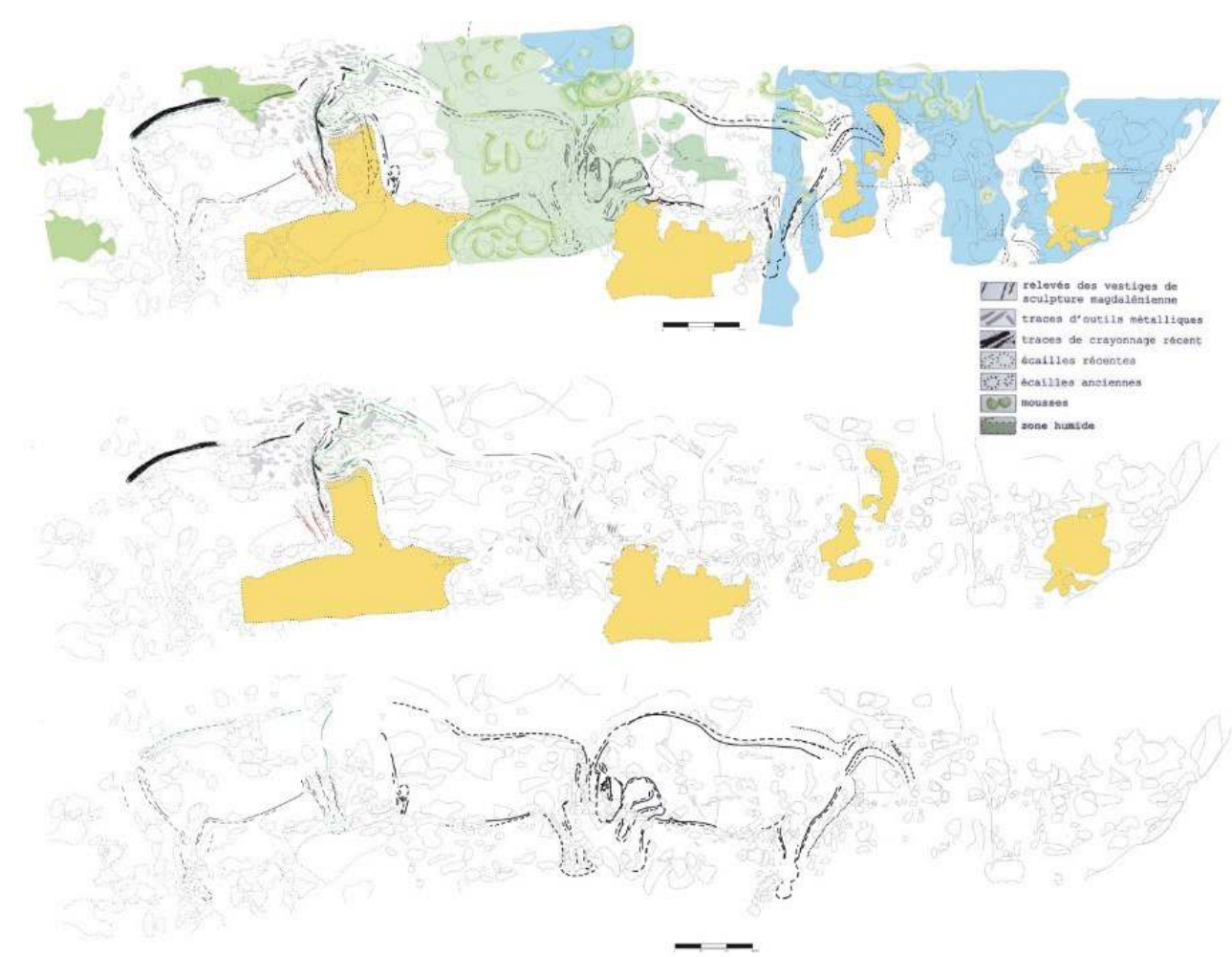

Relevé analytique et exhaustif par traitement vectoriel de la frise sculptée de la Chaire-à-Calvin. Chaque information est rassemblée au sein d'un calque (raster).

Document G. Pinçon, O. Fuentes, C. Bourdier, A. Abgrall, M. Peyroux, E. Bosch, 2008. (c) Geneviève Pinçon et. al.

La réalisation de ce type de relevé est subordonnée à l'association de multiples compétences. Ces « regards » et expertises, alliés au travail du préhistorien, permettent de rendre compte des richesses comme de la diversité des techniques et des supports des œuvres pariétales ou mobilières. Cette méthode s'enrichit donc du croisement des sciences et des spécialités. Cependant, du fait de leur aspect technique, la lecture des rendus graphiques n'est pas toujours aisée. Un travail d'organisation des données et de lisibilité du code graphique est alors nécessaire. L'environnement logiciel se base sur un traitement vectoriel sous licence Open Source (Inkscape) ou payante (OIllustrator).

\section{Horizons 3D et ère du numérique pour l'étude de l'art préhistorique}

Les besoins spécifiques aux différents intervenants (pouvoirs publics, collectivités, propriétaires, chercheurs, opérateurs, etc.) font que les usages et les finalités de la 3D peuvent diverger. La valorisation du patrimoine est un enjeu touristique et économique essentiel. Le modèle 3D peut être utilisé à des fins de médiation auprès du grand public et contribuer au développement touristique d'un territoire, à condition de consentir à l'investissement nécessaire. Seulement, ces actions liées à la protection et à la médiation ne peuvent réellement être de qualité et pertinentes que si le discours scientifique se trouve renouvelé. La 3D permet d'enrichir la documentation et apporte une contribution significative à la construction de la connaissance, comme le rappelle Serge Cassen : « Une 
première exigence est en effet de trouver les moyens d'obtenir une meilleure présentation des gravures conservées (pour Gavrinis en l'occurrence) ou perdues afin qu'une interprétation à leur propos puisse s'établir sur une base renouvelée ${ }^{18} . .$. ». Les technologies 3D permettent cette amélioration lorsque les outils sont utilisés pour répondre (en amont) à des problématiques scientifiques portant sur les programmes iconographiques paléolithiques ou post-paléolithiques.

\section{La grotte ornée de Comarque (Dordogne) comme cas d'école}

C'est dans cette continuité des cadres d'expérimentation technique et méthodologique que nous présentons les travaux réalisés au Centre national de préhistoire (CNP) associant Julie Lepelé, infographiste 3D en apprentissage au CNP et Oscar Fuentes, préhistorien, prestataire. Ils s'appuient sur le relevé tridimensionnel des parois de la grotte de Comarque effectué en 2015 par la société Art Graphique et Patrimoine ${ }^{19}$. Cette numérisation avait pour objectif de fournir une ressource pour l'étude de la cavité, un état de conservation de celle-ci et une base pour des projets de valorisation de la grotte ornée par le propriétaire qui exploite déjà le castrum de Comarque.

L'acquisition numérique a été réalisée au moyen d'un scan laser 3D Faro (scan rotatif $360^{\circ}$ ) avec une précision d'ensemble de $2 \mathrm{~mm}$ à $330 \mathrm{~m}$ pour la totalité de la cavité, et d'une précision de $0,5 \mathrm{~mm}$ pour les panneaux ornés à distance humaine. Une vidéogrammétrie a été réalisée avec un appareil Mantis (pied vidéo avec capteur). Cette acquisition a été réalisée avec une précision de moins de $0,5 \mathrm{~mm}$ et a permis une capture rapide.

\section{Modèle numérique : support du relevé d'art paléolithique}

Plusieurs logiciels ont été testés pour traiter les données numériques 3D (notamment Meshlab et Cloud Compare). Dans cet article, nous présentons exclusivement les applications que nous avons utilisées sous interface Blender ${ }^{20}$. Il s'agit d'un logiciel Open Source et collaboratif. À l'origine, ce logiciel a été conçu pour le domaine de la création graphique (permettant la modélisation, l'animation vidéo et les effets spéciaux). Contrairement à Meshlab ou à Cloud Compare, il offre un large panel de fonctionnalités très puissantes, qu'il est possible de détourner pour répondre aux nécessités qu'implique l'étude de l'art paléolithique. Il présente l'avantage de bénéficier de l'apport continuel de la communauté d'utilisateurs pour développer et compléter ses fonctionnalités. Il a donc été possible de faire appel aux contributeurs pour trouver une solution à chaque besoin. Chacun peut accéder aux sources des programmes, les adapter et partager ses nouveaux développements en les redistribuant dans la communauté ${ }^{21}$. Bien que d'une utilisation complexe, ce logiciel flexible offre une gamme d'applications permettant de procéder à une étude des représentations de qualité, le tout dans un environnement 3D.

Il est intéressant d'étudier dans quelle mesure la 3D mise en œuvre au moyen du logiciel Blender permet d'analyser les œuvres et d'effectuer des relevés d'analyse scientifique. L'exploitation des fichiers HD à travers ce logiciel a ainsi été à l'origine d'une série d'actions que nous présentons ici (fig. 5). 
Figure 5

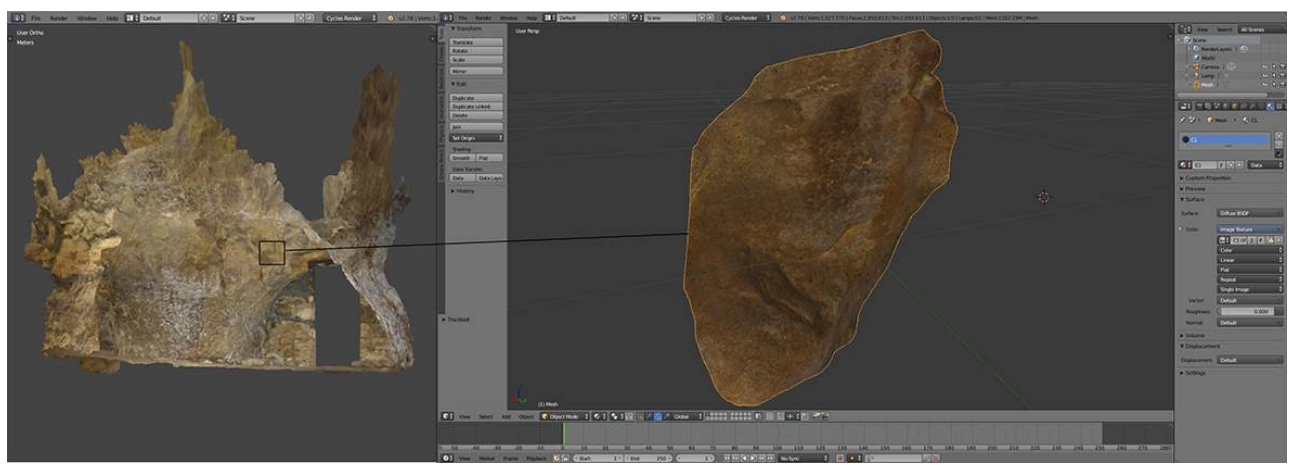

Ressources 3D utilisées : fichier « Grotte Complète », paroi droite à gauche de la Galerie Centrale ; fichier « Détails », détail du cheval n004 situé à droite.

Sources 3D : AGP/CNP, document : Fuentes et Lepelé. (c) Ministère de la Culture, Centre national de préhistoire.

Il s'est avéré essentiel de décomposer les démarches préalables au relevé afin de les transposer dans l'environnement 3D et de reproduire les étapes de travail qui sont normalement réalisées sur l'objet étudié. Par la suite, nous avons cherché à enrichir ce processus en proposant des applications et traitements numériques des données.

\section{La lumière}

L'une des premières étapes est la mise en lumière des œuvres pour obtenir des documents d'analyse. Nous avons utilisé les spots virtuels proposés par Blender avec différents paramètres : intensité, taille, dureté du contour. Cela a permis, à Comarque, par exemple, d'obtenir une image complète bien éclairée et en taille réelle du grand cheval sculpté en dépit de sa localisation dans la partie la plus exiguë de la cavité (0,80 $\mathrm{m}$ de recul) (fig. 6).

\section{Figure 6}

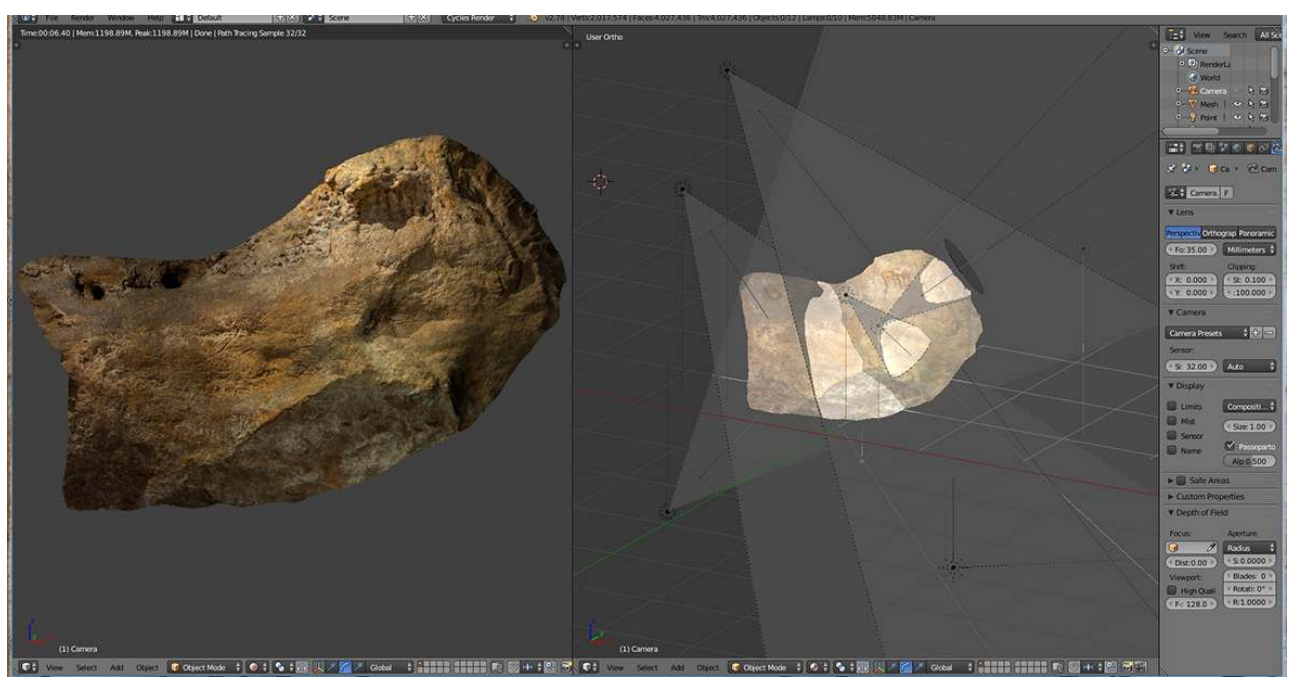

Utilisation des éclairages virtuels sous Blender : mise en lumière du cheval de Comarque. À gauche, la disposition des sources lumineuses, leur rayonnement et leur direction, à droite, le résultat obtenu. Sources 3D : AGP/CNP, document : Fuentes et Lepelé. ( ) Ministère de la Culture, Centre national de préhistoire. 


\section{Ortho-photographie 3D}

31 Les éclairages placés, il est alors possible de prendre un cliché en se servant de la caméra virtuelle. Grâce aux coordonnées de position et d'orientation attribuées à tous les objets 3D, nous réalisons un cliché ortho-plan en réglant le plan de la caméra en rapport à celui de la paroi. Pour obtenir une définition optimale de ce cliché, nous étudions la densité de points du maillage et la définition de la texture de la paroi. Ce cliché haute définition (en moyenne 7680 par 4320 pixels) sert de support pour la réalisation du relevé.

Pour compléter les informations recueillies grâce aux mises en lumière, nous avons cherché à tirer profit de la nature mathématique de l'objet 3D en mettant en place des traitements colorimétriques automatisés, s'appuyant sur les informations géométriques $\mathrm{du}$ mesh (maillage). Nous avons mis en place trois types de traitements (Pointiness, Normales et Courbes de Niveau). Ceux-ci procèdent de l'approche multimodale de la surface.

\section{Pointiness}

Il s'agit d'un traitement permettant de sélectionner les zones où l'angle entre plusieurs faces est faible. Cela permet de rendre visibles les zones de «creux» en leur attribuant une couleur. On obtient alors une cartographie des reliefs. Le "Pointiness» est une méthode « objective » dans le sens où elle rend tous les reliefs visibles. Il cartographie donc des lignes qu'on peut interpréter comme des fonds de traits. Reste à l'archéologue d'analyser ces informations et de les interpréter (fig. 7).

Figure 7

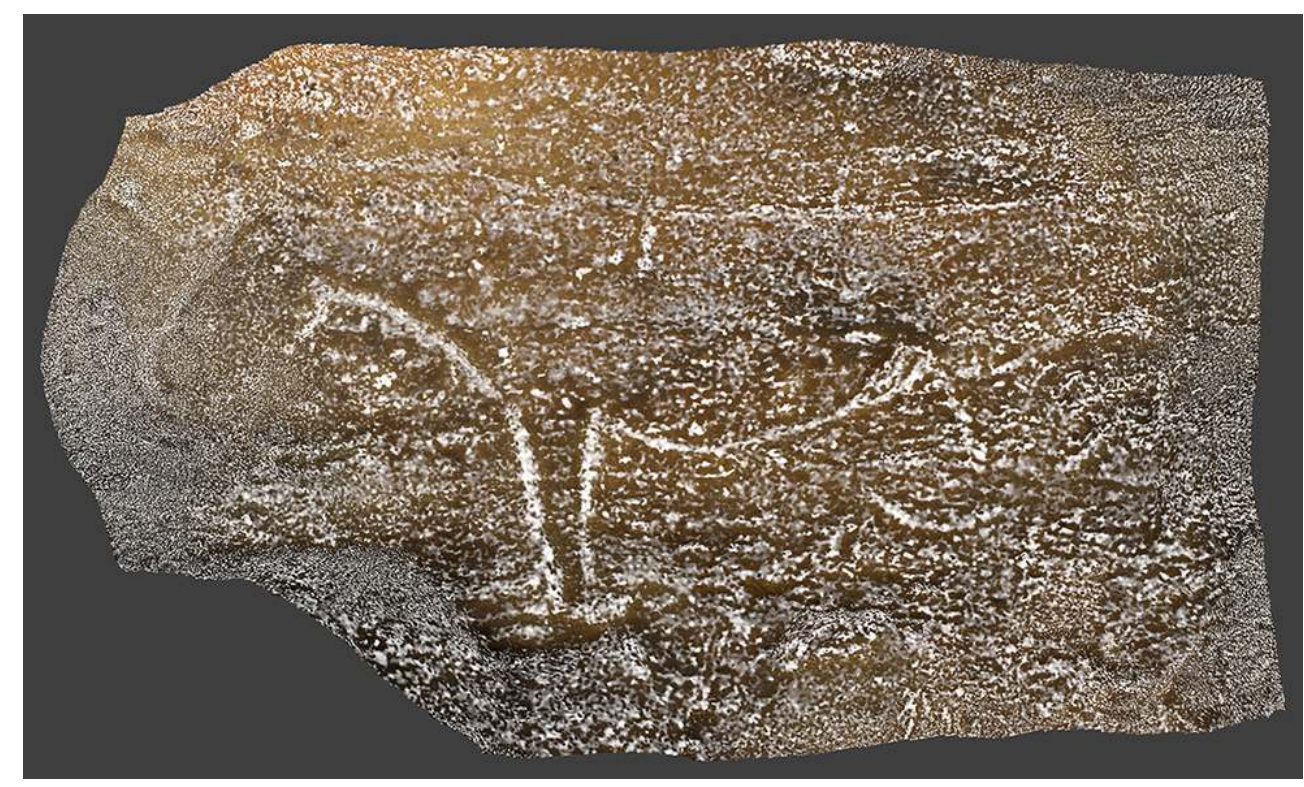

Traitement colorimétrique et cartographie de la surface par l'application « pointiness ». Cheval n²0, grotte de Comarque.

Sources 3D : AGP/CNP, document : Fuentes et Lepelé. (C) Ministère de la Culture, Centre national de préhistoire. 


\section{Normales}

Sur le modèle 3D, chaque face du maillage est caractérisée par sa " normale », c'est-à-dire une demi-droite perpendiculaire à cette face qui définit son sens intérieur/extérieur. Un traitement sur ces « normales » permet de colorer la paroi en fonction de l'orientation de celles-ci. En choisissant deux couleurs qui représentent deux orientations opposées, il est possible de cartographier les reliefs par un contraste entre les deux «versants» des volumes (fig. 8).

Figure 8

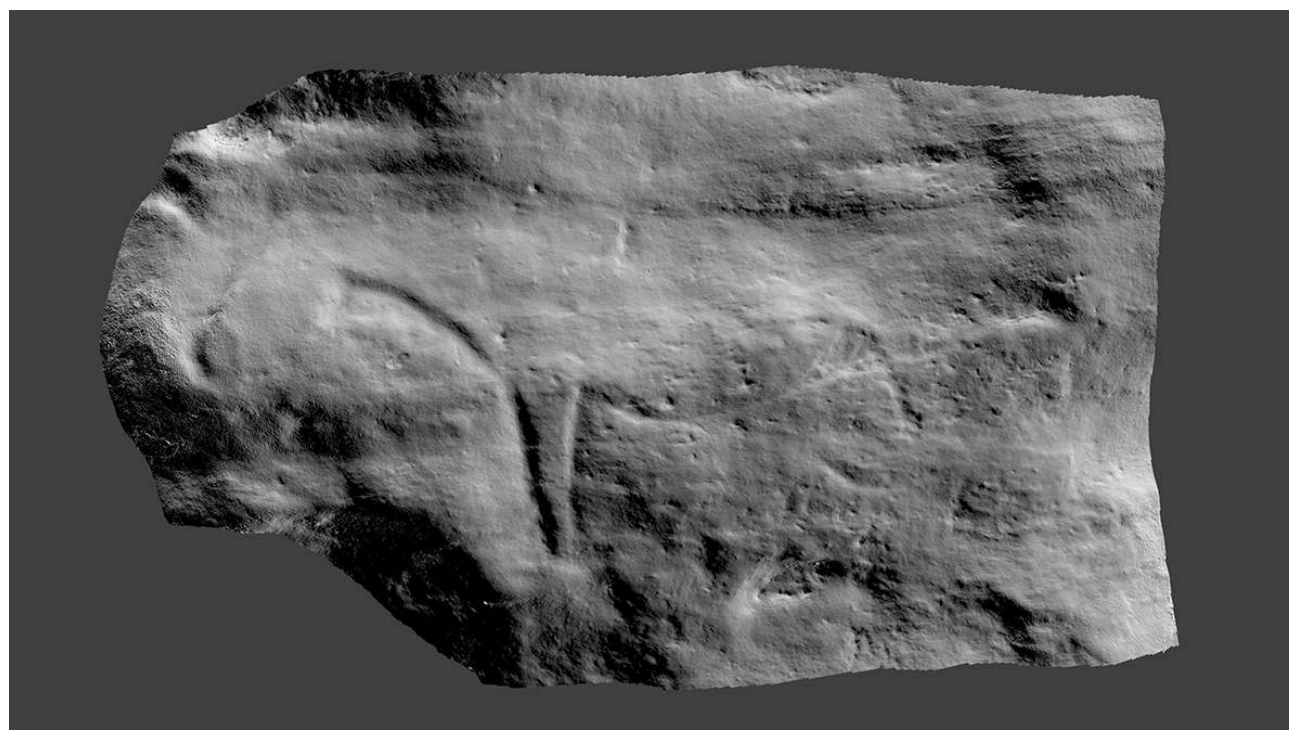

Traitement colorimétrique et cartographie de la surface par l'application « normales ». Cheval n²0, grotte de Comarque.

Sources 3D : AGP/CNP, document : Fuentes et Lepelé. (c) Ministère de la Culture, Centre national de préhistoire.

\section{Courbes de niveau}

Enfin, une troisième cartographie des surfaces peut être produite et enrichir la panoplie des calques pour le relevé, l'attribution de courbes de niveau. La 3D, comme nous l'avons $\mathrm{vu}$, permet de documenter le volume, et la production de ces courbes est un autre moyen cartographique de traduire la morphologie des surfaces dans le cadre d'un relevé (fig. 9). 


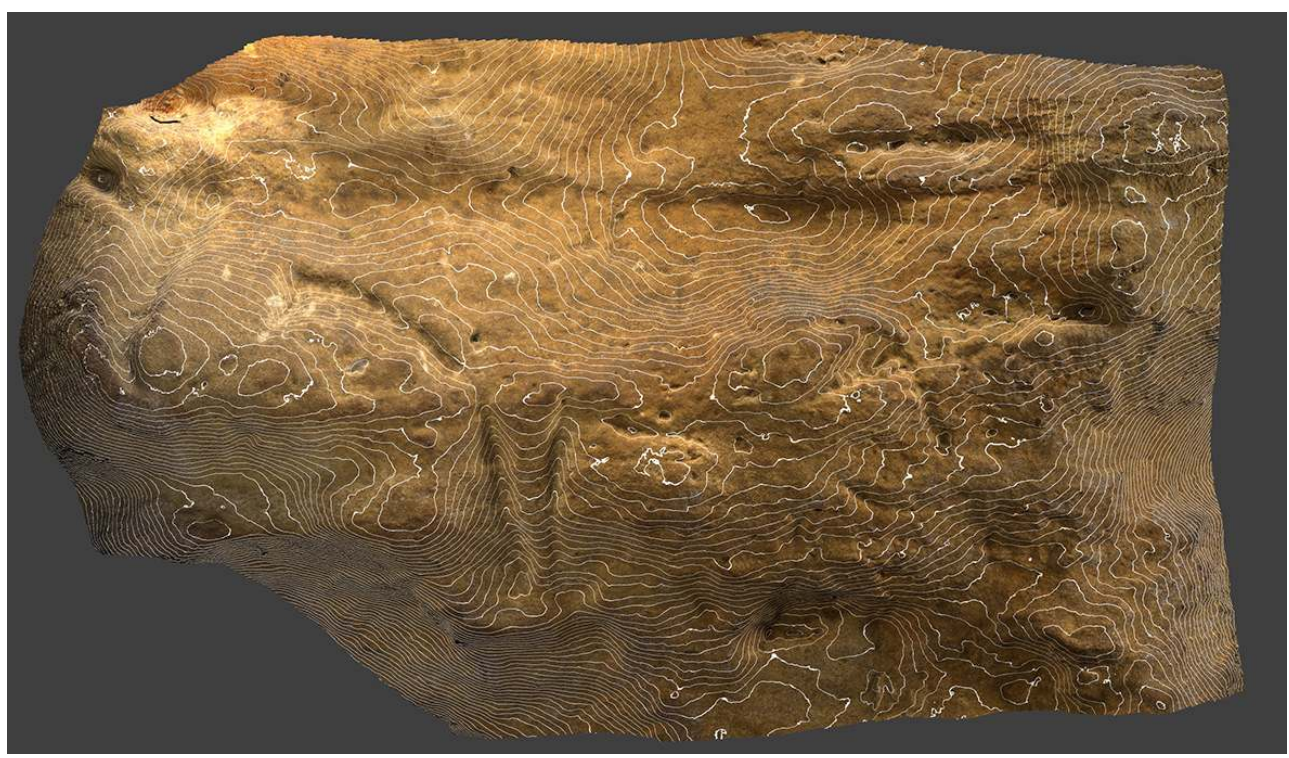

Traitement des surfaces et topographie des volumes par courbe de niveau. Cheval n²0, grotte de Comarque.

Sources 3D : AGP/CNP, document : Fuentes et Lepelé. (c) Ministère de la Culture, Centre national de préhistoire.

Ces trois traitements sont réalisés sur le même modèle 3D de la paroi. Étant donné que le modèle est fixe et que la caméra virtuelle a été paramétrée et posée de manière orthogonale, l'ensemble des traitements réalisés sont superposables et reproductibles.

\section{Prises de mesures}

Il est important d'accompagner l'étude des représentations graphiques de données métriques. Ces données aident à la fois à la compréhension des modes de réalisation des œuvres par les paléolithiques, et à générer des bases de données avec les informations liées à chaque image pour une mise en perspective des observations. Elles servent donc à enregistrer l'état conservatoire à un instant donné, mais aussi à mesurer ses états dans le temps, à estimer par exemple les altérations ou déperditions d'information.

La scène 3D peut être augmentée d'éléments de mesure informant sur le placement de caméra, par exemple. Ces données peuvent alors être comprises comme des métadonnées renseignant sur l'angle de la prise de vue, la distance de la caméra. Autant de données qui, d'une part, permettent de comprendre comment un tel résultat a été obtenu, et d'autre part, de reproduire la manipulation. Pour cette application, nous avons utilisé l' add-on « Measure It $»^{22}$.

\section{Écosystème 3D et relevé d'art paléolithique : une approche multimodale}

L'ensemble des traitements et l'approche multimodale peuvent alors être perçus comme une amorce de protocole des usages 3D dans l'analyse des images paléolithiques. Le préalable reste une bonne connaissance des lieux et des parois ou des supports par 
l'archéologue. Tout en tenant compte des éléments de déformation propres aux méthodes d'acquisition 3D, il est possible de constituer un écosystème de travail étendu susceptible de générer d'autres éléments informatifs. Il faut alors concevoir, simultanément, la méthodologie d'approche répondant à une problématique scientifique et la capacité de générer et gérer les métadonnées ainsi que les paradonnées.

À partir de l'exemple de la grotte de Comarque, nous proposons ici un protocole de relevé des surfaces permettant des transferts 2D sur 3D.

\section{Relevé 2D d'art paléolithique : transferts 2D/3D}

Il s'agit ici de la proposition d'une approche multimodale 3D s'appuyant sur la compilation de couches vectorielles (apparentées à des rasters), chacune relevant des informations différentes. Le préalable est la réalisation d'un relevé selon le cadre de référence relatif au "relevé technique", sous une interface vectorielle. En effet, la méthode proposée se base sur le transfert dans la scène 3D des couches vectorielles du relevé réalisé en 2D sous environnement vectoriel (fig. 10).

Figure 10

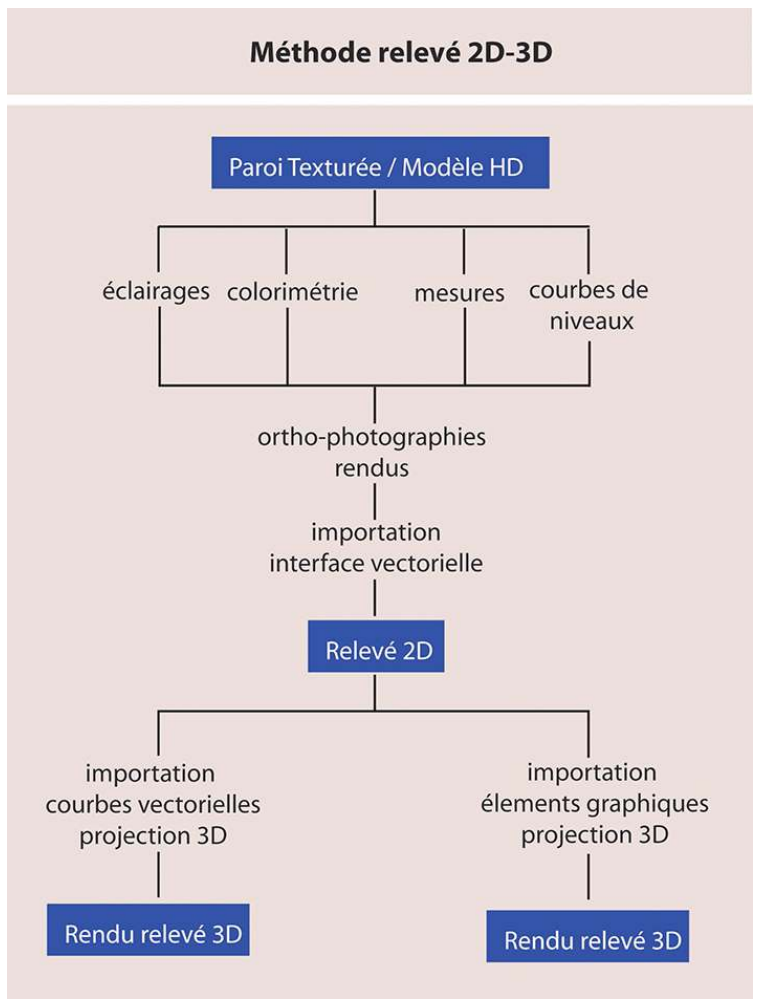

Étapes du relevé d'art paléolithique : transferts techniques 2D-3D. (c) Ministère de la Culture, Centre national de préhistoire.

Une fois l'ensemble des calques réalisés sous forme de couches vectorielles, il est possible, à partir du cliché ortho-plan obtenu dans Blender, de transférer ces informations graphiques dans le logiciel 3D, et de les projeter sur la surface du modèle de la paroi (fig. 11). 
Figure 11
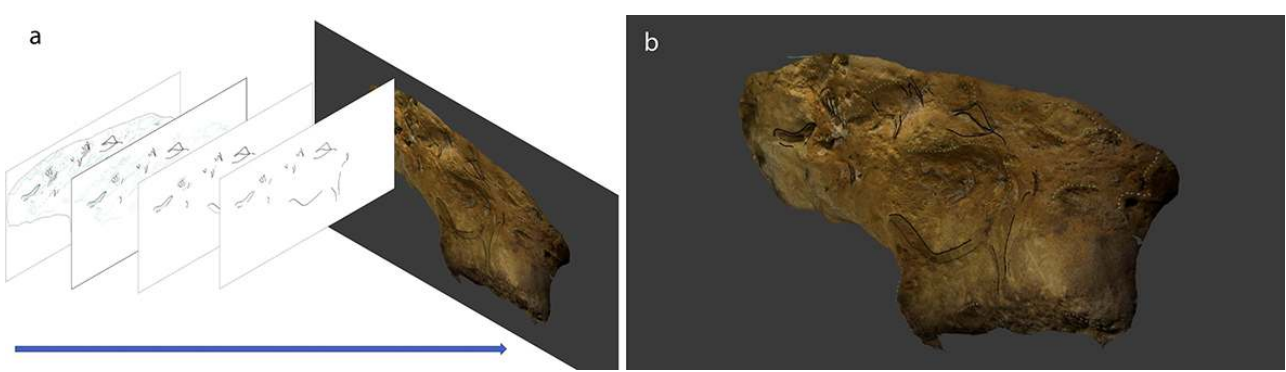

a) Transfert des calques raster.svg issus du relevé 2D sur le modèle $3 D$; b) Visualisation de l'intégration complète du relevé vectoriel 2D sur le modèle 3D.

Sources 3D : AGP/CNP, document : Fuentes et Lepelé. (c) Ministère de la Culture, Centre national de préhistoire.

Cependant, certaines limites d'exportation des fichiers vectoriels vers les fichiers 3D ont amené à déterminer deux méthodes, dont chacune présente des avantages et des inconvénients. En effet, lors de l'importation des relevés 2D sous forme de courbes de Bézier, les motifs de la charte graphique d'origine sont perdus, et s'avèrent complexes et pénibles à réaliser dans Blender. Nous pouvons alors appliquer une charte graphique simplifiée et conserver les propriétés d'édition dynamique des courbes.

L'autre méthode consiste à transformer initialement les courbes de Bézier en image vectorielle puis de les importer dans Blender. Cette méthode permet de conserver la complexité de la charte graphique mais entraîne la perte des propriétés éditables des courbes de Bézier. Une adaptation de la charte graphique à l'environnement 3D serait donc nécessaire à terme.

Grâce à ces méthodes, les relevés obtenus tirent vraiment profit des ressources 3D et restent manipulables et observables dynamiquement. Pourtant, le relevé serait plus juste s'il était réalisé directement sur le modèle 3D plutôt que d'après une image en 2D. C'est pourquoi nous exploitons les potentialités du logiciel Blender pour proposer un workflow (processus) de relevé ou système d'annotation directement en 3D.

\section{Relevé 3D d'art paléolithique : la démarche intégrale}

Pour la réalisation d'un relevé directement en 3D, nous utilisons le modèle sur lequel nous appliquons une nouvelle texture, obtenue par la technique du «Baking». Cette technique consiste à fixer les éclairages sur la surface et ainsi, faciliter la manipulation du modèle « mappé ».

Sur ce support, l'archéologue utilise le «Grease Pencil» («crayon gras») sous Blender pour commencer le relevé 3D. Il peut organiser les informations selon des systèmes de calques également en 3D. Tout comme dans un processus de relevé de type technique vectoriel, il est possible de regrouper l'information graphique déterminée par l'archéologue et de trier les ensembles d'éléments par calque (anthropique ancien, récent, état de surface, reliefs naturels, dépôts naturels, etc.) (fig. 12). 
Figure 12

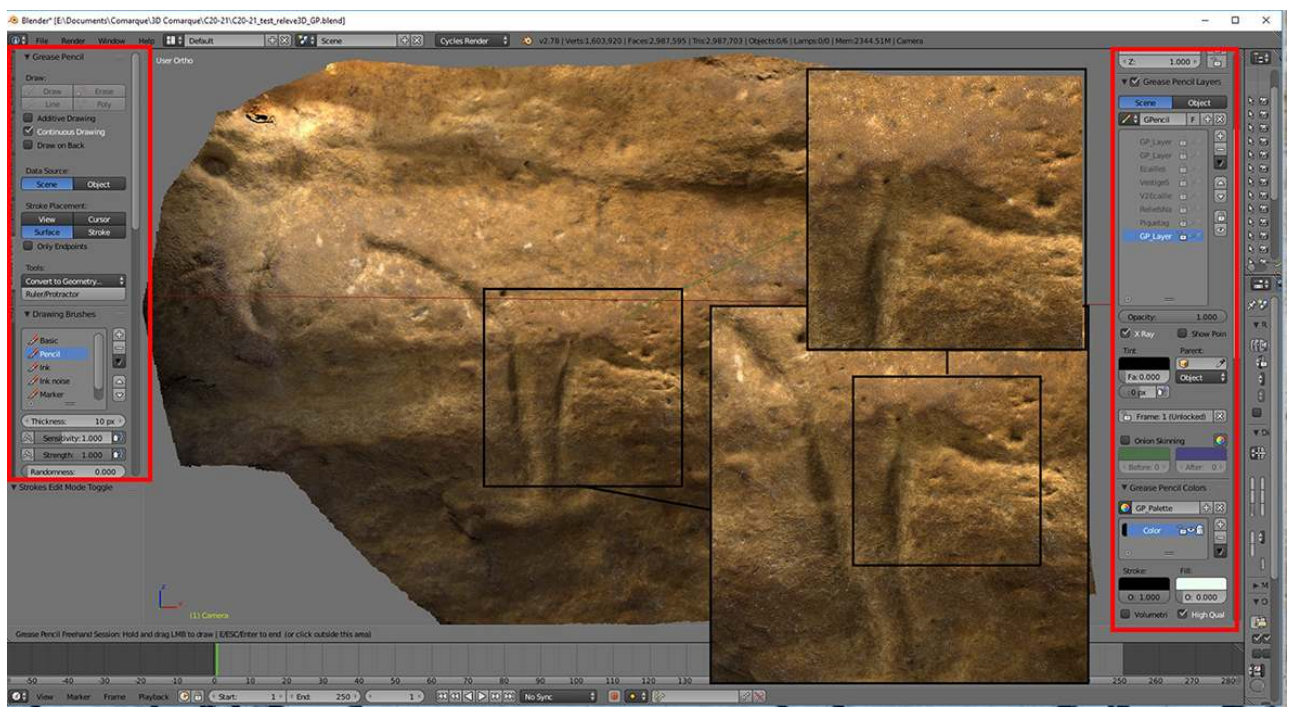

Interface Blender, utilisation du « Grease Pencil » pour la réalisation d'annotations sur le modèle 3D. À gauche, les paramétrages du « Grease Pencil », à droite, les calques et attributions (couleur, etc.). Un modèle de haute définition permet une « scène » de travail autorisant les agrandissements sur les détails de la paroi.

Sources 3D : AGP/CNP; document : Fuentes et Lepelé. @ Ministère de la Culture, Centre national de préhistoire.

L'étape suivante consiste à transformer les traits dessinés en éléments vectoriels, sous forme de courbes de Bézier (pouvant être modifiées).

Ce type de courbes vectorielles créées dans Blender s'apparente à celles que l'on importe depuis les environnements vectoriels 2D. Nous sommes donc confrontés au même cas de figure, et les éléments du relevé sont donc pour l'instant présentés dans une charte graphique simplifiée.

50 Cette méthode permet d'obtenir un relevé complètement réalisé sous environnement 3D, ou peut être utilisée comme un système d'annotation permettant, par exemple, de préparer le terrain ou le relevé à venir (pointer des informations, proposer des interprétations en attendant leur validation sur l'objet étudié) (fig. 13). 
Figure 13

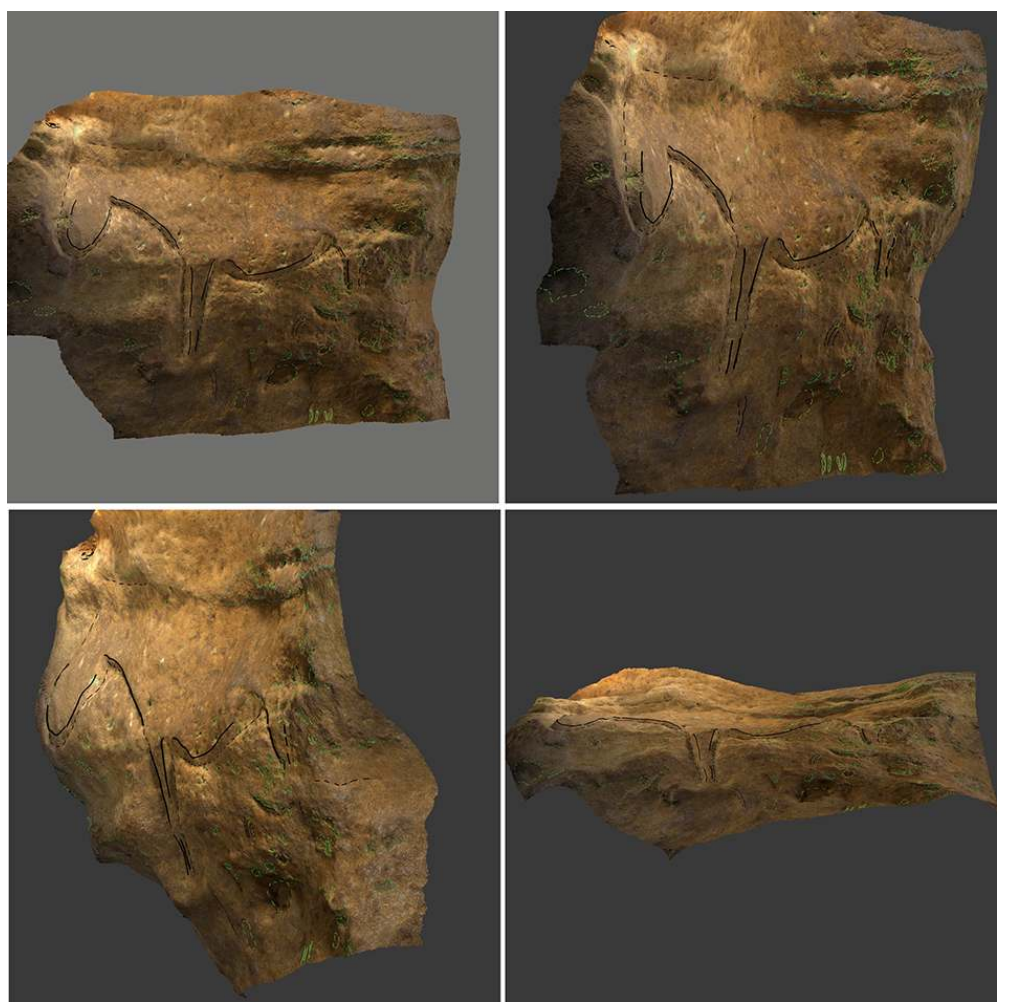

Relevé 3D réalisé sous Blender, cheval n²1, grotte de Comarque.

Sources 3D : AGP/CNP, document: Fuentes et Lepelé. (C) Ministère de la Culture, Centre national de préhistoire.

51 Dans ce workflow, il n'est plus question de transfert d'un environnement 2D vers la 3D puisque toutes les annotations sémantiques et graphiques se font directement dans le modèle 3D, de manière dynamique et en volume (fig. 14). 
Figure 14

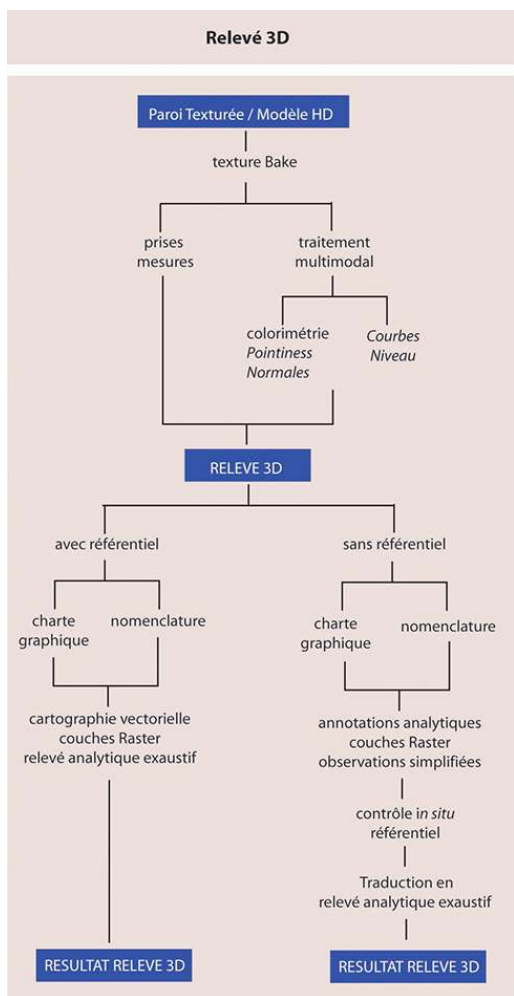

Schéma analytique du relevé 3D réalisé sous le système Blender, cadre de référence vectoriel analytique.

Sources 3D : AGP/CNP, document : Fuentes et Lepelé. (C) Ministère de la Culture, Centre national de préhistoire.

52 L'avantage de disposer de couches vectorielles, sous la forme de courbes de Bézier, est que les informations retranscrites peuvent être modifiées a posteriori, augmentées avec de nouvelles informations ou corrigées dans le cadre de changement d'identification des stigmates. Ces calques constituent une base de données évolutive, contrairement aux images de type « bitmap » qui sont figées (fig. 15). 


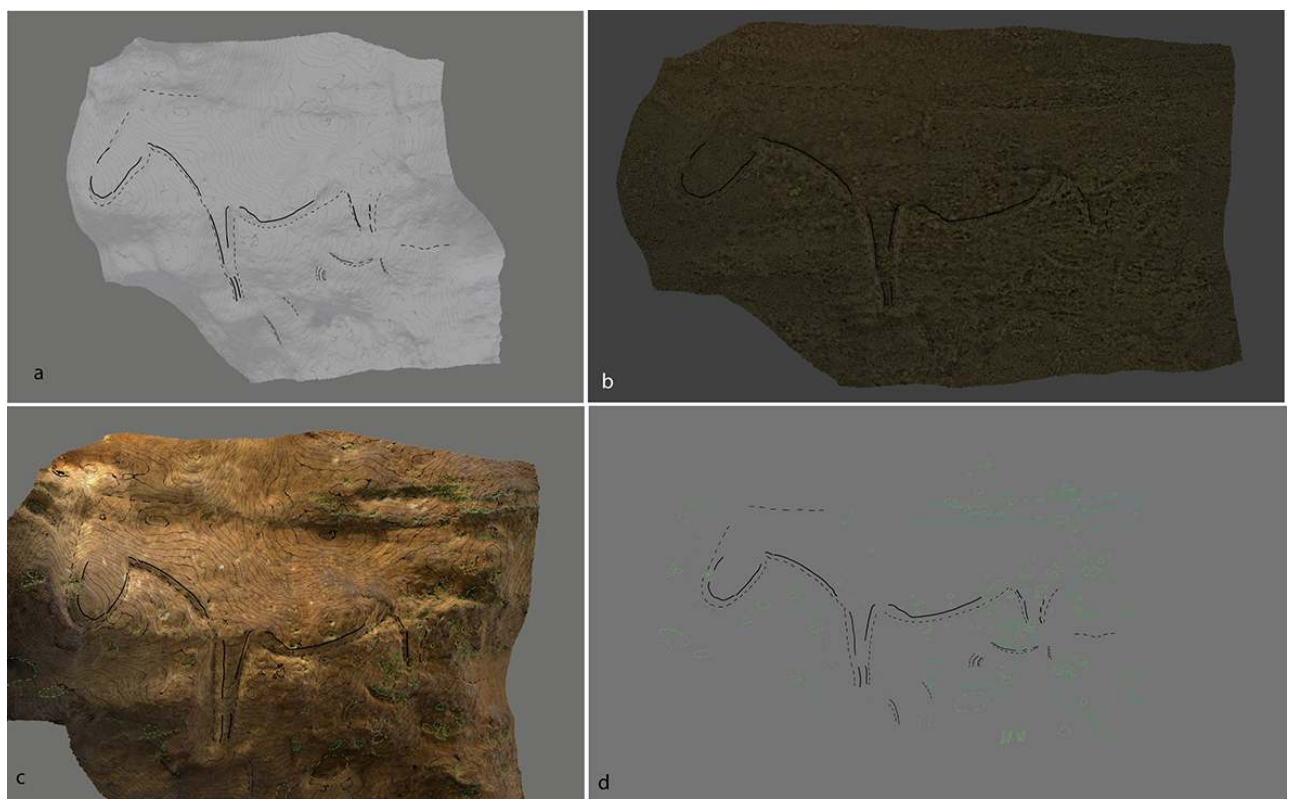

Visualisation multimodale du relevé 3D réalisé sous Blender : a) rendu du relevé vectoriel 3D avec Normales et Courbes de Niveau; b) relevé vectoriel sélectif (cheval) avec visualisation des Pointiness; c) relevé vectoriel 3D avec visualisation de l'image texturée Bake augmentée des Courbes de Niveau; d) relevé vectoriel 3D sans affichage du Mesh (texture).

Sources 3D : AGP/CNP, document : Fuentes et Lepelé. (c) Ministère de la Culture, Centre national de préhistoire.

\section{Conclusion}

53 Ce travail réalisé avec les ressources numériques 3D avait pour objectif de tester les apports et limites des écosystèmes 3D employés. Nous les avons appliqués sur la 3D de la grotte de Commarque à titre d'exemple.

Dans le cas d'une accessibilité restreinte au site ou aux objets d'art mobilier paléolithiques pour des raisons conservatoires et grâce à l'existence du modèle numérique, la recherche en art paléolithique n'est plus soumise à une perte d'information due au passage d'un objet d'étude en volume à des documents d'analyse en deux dimensions. La 3D permet de ne plus perdre cette troisième dimension, essentielle pour décrire la nature même des supports des œuvres : le relief. Cette dimension est encore plus essentielle lorsque l'on étudie des gravures ou des sculptures. Elle a également tout son intérêt dans un suivi de l'état de conservation de la paroi et des œuvres précises.

Les processus techniques et méthodologiques sont présentés sous deux formes distinctes, selon leur procédé, mais sont finalement interdépendants : un transfert des données 2D vers la 3D, ou un relevé totalement réalisé sous interface 3D. Dans les deux cas, l'objectif est d'adopter une démarche fonctionnelle et répondant aux besoins de la connaissance. Ce travail a nécessité une rétrospective intéressante sur les modes opératoires du relevéz ${ }^{3}$. L'écosystème 3D est d'autant plus opérationnel que le cadre de référence mis en place pour l'étude des supports ornés s'appuie sur une interface vectorielle et donc sur un relevé analytique technique. Ainsi, il s'avère que la manière de se confronter aux environnements 3D dépend aussi du cadre de référence en place de l'archéologue. 

modèle doit être adaptée aux exigences d'analyse souhaitées et l'environnement logiciel manipulable ou rendu accessible par une formation ou un accompagnement. Bien que la 3D propose des applications qui permettent de transcender la nature des objets (grotte ornée ou objet d'art mobilier), une relation forte avec l'entité originale ne doit pas être oubliée. C'est en ayant une bonne connaissance du site ou de l'objet que la 3D gagne en pertinence, que ce soit dans la phase d'acquisition, celle du traitement ou de l'interprétation des données. répond à trois exigences : conserver, analyser et restituer. Pour analyser, il faut rendre au plus juste les éléments observés. Du calque direct aux modèles 3D en passant par la prise photographique numérique, tout a toujours été réalisé dans la perspective de rendre une image la plus juste possible. L'écosystème 3D doit se poser comme participant à la démarche du chercheur, à sa chaîne opératoire scientifique. Dans un protocole des usages de la 3D en lien avec les impératifs conservatoires des grottes ornées ou de l'art mobilier dans les musées, il convient désormais de normaliser les pratiques, ou du moins, de préciser un canevas technique et méthodologique qui puisse être partagé au-delà des cadres de référence propres à chaque équipe de recherche.

L'horizon 3D de demain nécessite une recherche collaborative et participative, orientée sur un plan horizontal et non plus vertical, favorisant l'interdisciplinarité et l'enrichissement mutuel sur un même objet patrimonial. La constitution des équipes de recherche dans le cadre d'une opération archéologique dans un site orné doit désormais prendre en compte la variété des compétences pour assurer la meilleure exploitation et la meilleure gestion possible de ces nouvelles ressources numériques.

\section{BIBLIOGRAPHIE}

ABGRALL, Aurélie, BOURDIER, Camille, FUENTES, Oscar, PINÇON, Geneviève. « Le relevé graphique analytique : un nouvel outil pour l'étude des œuvres d'art paléolithique ». Dans BUISSON-CATIL, Jacques, PRIMAULT, Jérôme (dir.). Préhistoire entre Vienne et Charentes. Hommes et sociétés du Paléolithique. Chauvigny: Association des publications chauvinoises, coll. « Mémoire. Société de recherches archéologiques de Chauvigny, 38 ", 2010, p. 476-484.

ARCHAMBEAU, Monique. Les Figurations humaines pariétales périgourdines. Étude d'un cas : Les Combarelles. Thèse de doctorat de troisième cycle. Aix-Marseille : université de Provence, 1984.

AZÉMA, M., GÉLY, B., PRUDHOMME, Fr., ATM 3D. « Relevé 3D de gravures fines paléolithiques dans l'abri du Colombier (gorges de l'Ardèche) », In Situ [En ligne], 13 | 2010, mis en ligne le 16 avril 2012, consulté le 26 février 2018. URL : http://journals.openedition.org/insitu/6723 ; DOI : 10.4000/insitu.6723.

AZÉMA, Marc, GÉLY, Bernard, BOURILLON, Raphaëlle, LHOMME, David. « La grotte ornée paléolithique de Baume Latrone (France, Gard) : la 3D remonte le temps... ». Dans CLOTTES, Jean (éd.). L'Art pléistocène dans le monde / Pleistocene art of the world / Arte pleistoceno en el mundo. Actes 
du congrès IFRAO, Tarascon-sur-Ariège, septembre 2010 - Symposium « Application des techniques forensiques aux recherches sur l'art pléistocène. Société Préhistorique Ariège Pyrénées. Préhistoire, Art et Société, 2010-2011, t. 65-66, p. 1221-1238.

BOURDIER, Camille. « La frise sculptée de l'abri Reverdit (Sergeac, Dordogne) : première approche analytique des œuvres ». Paléo, 2008, 20, p. 23-46.

BOURDIER, Camille, ABGRALL, Aurélie, HUARD, Olivier, LE BRUN, Éric, PEYROUX, Magali, PINÇON, Geneviève. «Histoires de bisons et de chevaux : regard sur l'évolution de la frise pariétale du Cap-Blanc (Marquay, Dordogne) à travers l'analyse du panneau de l'alcôve ». Paléo, 2009-2010, 21, p. 17-38.

CASSEN, Serge, GRIMAUD, Valentin, LESCOP, Laurent, ROBINET, Elven, MARCOUX, Nancy. «Étude sur un monolithe - la dalle S12 au sol du monument de Gavrinis (Larmor-Baden, Morbihan) ». Revue archéologique de l'Ouest, 2016, 33, p. 55-76.

FLICHY, Pascal. L'Innovation technique. Récents développements en sciences sociales. Vers une nouvelle théorie de l'innovation. Paris : La Découverte, 2003.

FRITZ, Carole, TOSELLO, Gilles. « The hidden meaning of forms : Methods of recording Paleolithic parietal art ». Journal of Archaeological Method and Theory, 2007, 14, 1, p. 48-80.

FRITZ, Carole, TOSELLO, Gilles, AZÉMA, Marc, MOREAU, O., PERAZIO, G., PERAL, G. « Restauration virtuelle de l'art pariétal paléolithique : le cas de la grotte de Marsoulas », In Situ [En ligne], 13 | 2010, mis en ligne le 16 avril 2012, consulté le 26 février 2018. URL : http:// journals.openedition.org/insitu/6774 ; DOI : 10.4000/insitu.6774.

FUENTES, Oscar, BOURDIER, Camille, FERRIER, Catherine, HUARD, Olivier, LANGLAIS, Mathieu, MEVEL, Ludovic. Grotte de Comarque (Eyzies-de-Tayac-Sireuil, Dordogne). Rapport intermédiaire d'opération archéologique, Prospection avec relevé d'art rupestre. SRA Aquitaine, 2015.

FUENTES, Oscar. « La 3D et l'étude de l'art pariétal, ses apports et ses limites. À quel moment le retour à l'original s'impose aux chercheurs? » Rapport d'étude. Périgueux : Centre national de préhistoire, 2017, $310 \mathrm{p}$.

IAKOVLEVA, Ludmila, PINÇON, Geneviève. Angles-sur-l'Anglin. La frise sculptée du Roc-aux-Sorciers. Paris : Réunion des musées nationaux/CTHS, 1997, 168 p.

LEROI-GOURHAN, André. Préhistoire de l'art occidental [1965]. Paris : Mazenod, 1978, 499 p.

LORBLANCHET, Michel, DELPECH, Françoise, RENAULT, Philippe, ANDRIEUX, Claude. « La grotte de Sainte-Eulalie à Espagnac (Lot) ». Gallia Préhistoire, 1973, 16, 1, p. 3-62

PINÇON, Geneviève. « L'art pariétal de la Chaîre-à-Calvin (Mouthiers-sur-Bohëme) ». Dans BUISSON-CATIL, J., PRIMAULT (éd.). Préhistoire entre Vienne et Charentes. Hommes et sociétés du Paléolithique. Publications Chauvignoises, 2010, XXXVIII, p. 461-475.

PINÇON, Geneviève, BOURDIER, Camille, FUENTES, Oscar. « Les sculptures pariétales magdaléniennes du Roc-aux-Sorciers (Vienne) et de la Chaire-à-Calvin (Charente) : œuvres d'un groupe culturel ou d'un seul et même artiste? ». Dans VERGNIEUX, Robert, DELEVOIE, Caroline (éd.). Virtual Retrospect 2007. Bordeaux : Ausonius, 2008, p. 13-20

PINÇON, Geneviève, BOURDIER, Camille, FUENTES, Oscar, ABGRALL, Aurélie, HAMON, Gaël. «Applications des technologies 3D à la sculpture pariétale magdalénienne. Lectures d'une iconographie reprise mais toujours renouvelée de deux abris sculptés du Magdalénien moyen grâce à l'usage de bibliothèques de formes 3D ». Dans PAILLET, Patrick (dir.). Les Arts de la Préhistoire : micro-analyses, mises en contextes et conservation, Paléo, $\mathrm{n}^{\circ}$ spécial, 2014, p. 85-92. 
TYMULA, Sophie. L'Art solutréen du Roc-de-Sers (Charente). Paris : Éd. de la Maison des sciences de l'homme, coll. « D.A.F. », 2002, 285 p.

\section{NOTES}

1. - PALES, Léon, TASSIN de SAINT-PÉREUSE, Marie. Les Gravures de la Marche. Bordeaux: impr. Delmas, 1969, p. 35.

2. - LEROI-GOURHAN, André. Préhistoire de l'art occidental [1965]. Paris : Mazenod, 1978, p. 240.

3. - FLICHY, Pascal. L'Innovation technique. Récents développements en sciences sociales. Vers une nouvelle théorie de l'innovation. Paris : La Découverte, 2003.

4. - FRITZ, Carole, TOSELLO, Gilles. "The hidden meaning of forms: Methods of recording Paleolithic parietal art ». Journal of Archaeological Method and Theory, 2007, 14, 1, p. 48-80, ici p. 61.

5. - Centre de recherche et d'étude pour l'art préhistorique. Cette plate-forme est rattachée administrativement à la Maison des sciences de l'homme et de la Société de Toulouse (USR 3414). 6. - Voir le site : http://www.creap.fr/methodes_releves.htm [consulté le 20/03/2019].

7. - FRITZ, Carole, TOSELLO, Gilles. Art. cit.

8. - AZÉMA, Marc, GÉLY, Bernard, BOURILLON, Raphaëlle, LHOMME, David. « La grotte ornée paléolithique de Baume Latrone (France, Gard) : la 3D remonte le temps... ». Dans CLOTTES, Jean (éd.). L'Art pléistocène dans le monde / Pleistocene art of the world / Arte pleistoceno en el mundo. Actes $\mathrm{du}$ congrès IFRAO, Tarascon-sur-Ariège, septembre 2010 - Symposium «Application des techniques forensiques aux recherches sur l'art pléistocène. Société Préhistorique Ariège Pyrénées. Préhistoire, Art et Société, 2010-2011, t. 65-66, p. 1221-1238, ici p. 1226.

9. - ABGRALL, Aurélie, BOURDIER, Camille, FUENTES, Oscar, PINÇON, Geneviève. «Le relevé graphique analytique: un nouvel outil pour l'étude des œuvres d'art paléolithique ». Dans BUISSON-CATIL, Jacques, PRIMAULT, Jérôme (dir.). Préhistoire entre Vienne et Charentes. Hommes et sociétés du Paléolithique. Chauvigny: Association des publications chauvinoises, coll. "Mémoire. Société de recherches archéologiques de Chauvigny, 38 », 2010, p. 476-484.

10. - LORBLANCHET, Michel, DELPECH, Françoise, RENAULT, Philippe, ANDRIEUX, Claude. «La grotte de Sainte-Eulalie à Espagnac (Lot) ». Gallia Préhistoire, 1973, 16, 1, p. 3-62 ; ARCHAMBEAU, Monique. Les Figurations humaines pariétales périgourdines. Étude d'un cas : Les Combarelles. Thèse de doctorat de troisième cycle. Aix-Marseille : université de Provence, 1984.

11. - IAKOVLEVA, Ludmila, PINÇON, Geneviève. Angles-sur-l'Anglin. La frise sculptée du Roc-auxSorciers. Paris : Réunion des musées nationaux/CTHS, 1997.

12. - PINÇON, Geneviève, BOURDIER, Camille, FUENTES, Oscar, ABGRALL, Aurélie, HAMON, Gaël. «Applications des technologies 3D à la sculpture pariétale magdalénienne. Lectures d'une iconographie reprise mais toujours renouvelée de deux abris sculptés du Magdalénien moyen grâce à l'usage de bibliothèques de formes 3D ». Dans PAILLET, Patrick (dir.). Les Arts de la Préhistoire : micro-analyses, mises en contextes et conservation, Paléo, $\mathrm{n}^{\circ}$ spécial, 2014, p. 85-92.

13. - PINÇON, Geneviève, BOURDIER, Camille, FUENTES, Oscar. «Les sculptures pariétales magdaléniennes du Roc-aux-Sorciers (Vienne) et de la Chaire-à-Calvin (Charente) : œuvres d'un groupe culturel ou d'un seul et même artiste? ». Dans VERGNIEUX, Robert, DELEVOIE, Caroline (éd.). Virtual Retrospect 2007. Bordeaux: Ausonius, 2008, p. 13-20 ; PINÇON, G., BOURDIER, C., FUENTES, O., ABGRALL, A., HAMON, G. « Applications des technologies 3D à la sculpture pariétale magdalénienne... ». Art. cit.

14. - TYMULA, Sophie. L'Art solutréen du Roc-de-Sers (Charente). Paris : Éd. de la Maison des sciences de l'homme, coll. « D.A.F. », 2002.

15. - BOURDIER, Camille. «La frise sculptée de l'abri Reverdit (Sergeac, Dordogne) : première approche analytique des œuvres ». Paléo, 2008, 20, p. 23-46. 
16. - BOURDIER, Camille, ABGRALL, Aurélie, HUARD, Olivier, LE BRUN, Éric, PEYROUX, Magali, PINÇON, Geneviève. "Histoires de bisons et de chevaux: regard sur l'évolution de la frise pariétale du Cap-Blanc (Marquay, Dordogne) à travers l'analyse du panneau de l'alcôve ». Paléo, 2009-2010, 21, p. 17-38.

17. - FUENTES, Oscar, BOURDIER, Camille, FERRIER, Catherine, HUARD, Olivier, LANGLAIS, Mathieu, MEVEL, Ludovic. Grotte de Comarque (Eyzies-de-Tayac-Sireuil, Dordogne). Rapport intermédiaire d'opération archéologique, Prospection avec relevé d'art rupestre. SRA Aquitaine, 2015.

18. - CASSEN, Serge, GRIMAUD, Valentin, LESCOP, Laurent, ROBINET, Elven, MARCOUX, Nancy. "Étude sur un monolithe - la dalle S12 au sol du monument de Gavrinis (Larmor-Baden, Morbihan) ». Revue archéologique de l'ouest, 2016, 33, p. 55-76; ici p. 14.

19. - Selon un cahier des charges précis réalisé par le CNP en concertation avec l'équipe scientifique en accord avec le propriétaire du site.

20. - Dans le cadre de ce travail nous avons utilisé la version 2.78 du logiciel Blender.

21. - Par exemple, un utilisateur français, sous le pseudonyme de Domlysz, a publié un programme permettant d'exploiter des données SIG au sein du logiciel, et de les traiter en 3D. Le programme, intitulé « Blender GIS », est disponible sur la plate-forme GitHub.

22. - Développé par un utilisateur sous le pseudonyme « Antonioya ».

23. - FUENTES, Oscar. « La 3D et l'étude de l'art pariétal, ses apports et ses limites. À quel moment le retour à l'original s'impose aux chercheurs? » Rapport d'étude. Périgueux : Centre national de préhistoire, 2017.

\section{RÉSUMÉS}

Toute étude portant sur l'art paléolithique (pariétal ou mobilier) s'appuie sur un relevé analytique qui se pratique depuis plus d'un siècle. Cette démarche d'analyse est indispensable à la compréhension de la pensée des sociétés préhistoriques. Les nouvelles technologies 3D bouleversent à nouveau les pratiques des chercheurs, comme cela a été le cas auparavant avec la photographie. À l'initiative du Centre national de préhistoire qui a sollicité un archéologue spécialiste de l'art pariétal et une infographiste 3D, un nouvel usage de la 3D appliquée à l'étude de l'art rupestre a pu être développé. Il permet de dépasser les limites inhérentes aux approches traditionnelles (relevés 2D). Les expressions graphiques peuvent enfin être étudiées dans leur contexte tridimensionnel. Nous avons choisi d'exploiter les ressources 3D pour faire dialoguer deux cadres de fonctionnement (2D-3D) que nous proposons d'illustrer dans cet article à partir de l'exemple de la grotte ornée de Comarque (Dordogne). Nous nous appuyons sur l'utilisation du logiciel Blender (Open Source) qui offre une panoplie d'outils aidant à l'analyse des œuvres pariétales. Il est notamment possible de projeter sur le modèle 3D des relevés vectoriels réalisés en 2D. Mais il est surtout possible de faire un relevé directement sur le modèle 3D en gardant les informations relatives au volume. Cette introduction des nouvelles technologies en 3D pour les relevés d'art rupestre permet aussi de renvoyer les archéologues aux questions méthodologiques sur lesquelles se basent leurs analyses.

All studies concerning Palaeolithic art (parietal or movable art) are based on analytical recordings of the kind now practiced for over a century. This analytical approach is indispensable for our understanding of the thought processes of prehistoric societies. But today, 
new 3D technologies are having a massive impact on research practices, as was the case with photography. An initiative by the Centre national de Préhistoire led to the development by an archaeologist specialising in parietal art, working in collaboration with a 3D-graphics designer, of a new way of applying 3D techniques to rock art. This makes it possible to go beyond the limits inherent in traditional approaches (2D recordings): graphic expressions can at last be studied in their three-dimensional context. We have chosen to use 3D resources in order to set up a dialogue between two operational frameworks (2D and 3D) which we propose to illustrate in this article, using the example of the decorated cave of Comarque (Dordogne). We base our approach on the use of Blender software (Open Source) which offers a panoply of tools useful for the analysis of parietal works. In particular, it is possible to project vectorial recordings made in 2D onto a 3D model. But above all it is possible to produce a record directly on the 3D model while keeping all the information relating to volume. This introduction of new 3D technologies for rock art recording may also encourage archaeologists to re-examine the methodological questions on which they base their analyses.

\section{INDEX}

Keywords : Upper Palaeolithic, prehistoric art, cave art, tracing, 3D laser scanning, vectorial tracing, methodology

Mots-clés : Paléolithique supérieur, art préhistorique, art pariétal, relevé, numérisation laser 3D, relevé vectoriel, méthodologie

\section{AUTEURS}

\section{OSCAR FUENTES}

Centre national de préhistoire, Ministère de la Culture - Direction générale des patrimoines sous-direction de l'archéologie oscar.fuentes@culture.gouv.fr

\section{JULIE LEPELÉ}

Infographiste 3D julie.lepele@outlook.fr

\section{GENEVIÈVE PINÇON}

Directrice du Centre national de préhistoire (CNP), Ministère de la Culture - direction générale des Patrimoines - sous-direction de l'Archéologie genevieve.pincon@culture.gouv.fr 